\title{
October 2013 Pulmonary Case of the Month: A Hidden Connection
}

\author{
Kelly Cawcutt, MD \\ Pritish Tosh, MD \\ Jennifer Elmer, RN, CNS \\ Scott Copeman, RRT \\ Christina Rivera, Pharm D, RPh \\ Division of Critical Care \\ Mayo Clinic \\ Rochester, Minnesota
}

History of Present IIIness

A 58 year old woman, former smoker, presented to the pulmonary outpatient clinic at Mayo Clinic Rochester with dyspnea on exertion. In clinic, she was found to be tachycardic and febrile, and therefore, she was directly admitted to a medicine ward for possible sepsis.

She had progressive dyspnea on exertion, accompanied by symptoms of dry cough, muscle weakness, dry mouth, easy bruising and constipation without weight loss for approximately 9 months. During this time, she was also diagnosed with an idiopathic pulmonary embolus with initiation of warfarin.

\section{$\mathrm{PMH}, \mathrm{SH}, \mathrm{FH}$}

During an extensive work-up for these symptoms she was found to have a Ca2+ channel antibody, with concern raised for possible paraneoplastic etiology, as positron emission tomography (PET) imaging also revealed abnormal uptake in lungs along with multiple lymph nodes, pancreatic tail, decreased cerebral metabolism suggestive of a diffuse encephalopathy and bilateral pulmonary infiltrates with cavitation in the lingula. She was also noted to have anemia and thrombocytopenia. Of note, she was up-to-date on all recommended cancer screenings.

\section{Physical Examination}

The patient was febrile $\left(39^{\circ} \mathrm{C}\right)$, tachypneic (30 breaths $\left./ \mathrm{min}\right)$ and tachycardic $(110$ beats/min) but blood pressure was normal $(110 / 68 \mathrm{~mm} \mathrm{Hg})$. Otherwise physical examination was unremarkable.

\section{Laboratory}

CBC: Hemoglobin $9.4 \mathrm{~g} / \mathrm{dL}$, white blood cell count 6,200 cells $/ \mathrm{mcL}$, platelet count $45,000 / \mathrm{mcl}$

Lactate $1.8 \mathrm{mmol} / \mathrm{L}$ 
INR: 2.1

\section{Radiography}

Admission chest $x$-ray is shown in figure 1 and the PET scan obtained prior to admission in figure 2 .

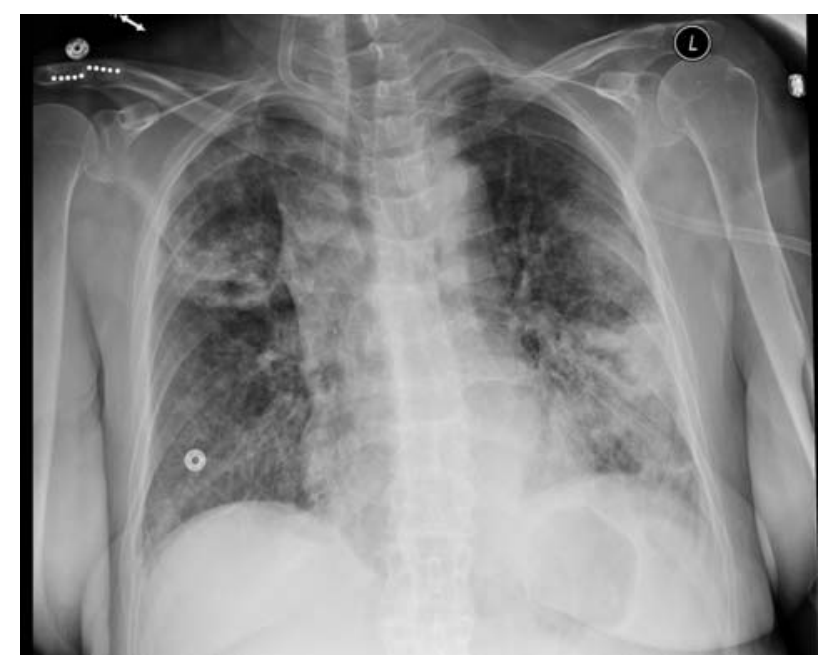

Figure 1. Admission chest x-ray.
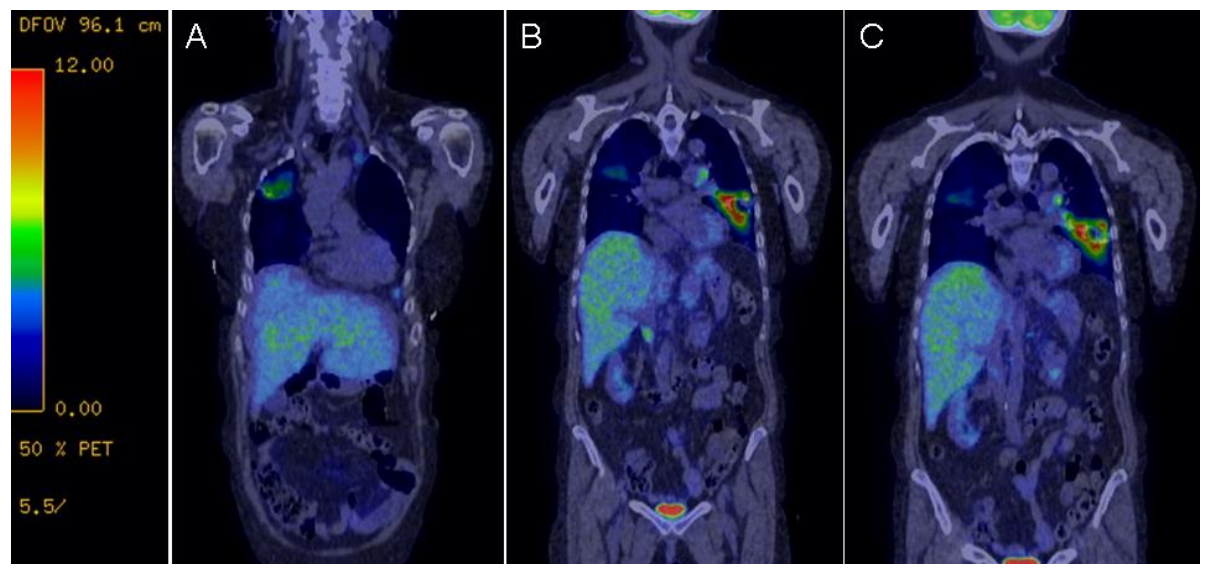

Figure 2. Representative coronal images of the PET scan obtained prior to admission showing abnormal uptake in lungs along with multiple lymph nodes, pancreatic tail, decreased cerebral metabolism suggestive of a diffuse encephalopathy and bilateral pulmonary infiltrates with cavitation in the lingula.

Which of the following should be done on admission?

1. Blood culture, sputum culture and urine culture

2. Broad spectrum antibiotic coverage

3. Intravenous fluids

4. Urine culture

5. All of the above 


\section{Correct! \\ 5. All of the above}

The patient is apparently acutely ill. The source of the illness is unclear but the fever with tachypnea, tachycardia and chest $x$-ray suggest possible pneumonia with the possibility of sepsis. Urine cultures are indicated since urinary tract infections are a common source of infection and sepsis in adults. Although her blood pressure is well preserved and her lactate is normal, her clinical picture suggests a high possibility of intravascular volume depletion and fluids are appropriate. The severity of her illness suggests that empiric broad spectrum antibiotics are indicated. She was begun on vancomycin, cefepime and levofloxacin.

The initial Gram stain of her sputum and all bacterial cultures were negative.

To better define her thoracic process a CT scan was performed (Figure 3).

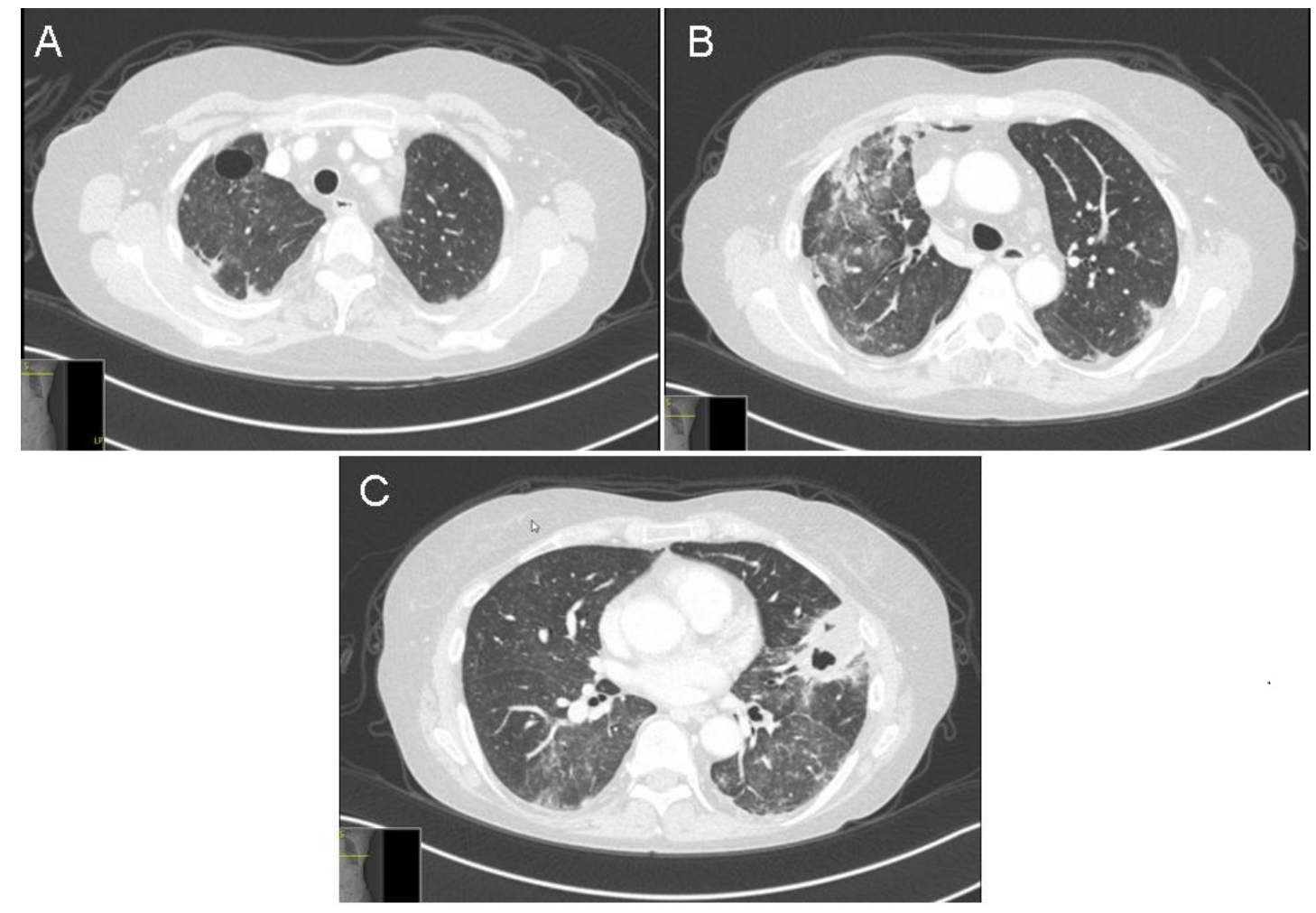

Figure 3. Representative images from admission thoracic CT scan.

Which of the following laboratory testing is least likely to be positive?

1. Acid fast smear and tuberculosis culture of sputum

2. Influenza polymerase chain reaction (PCR) from nasal swab

3. Serum coccidioidomycosis antibody

4. Urine histoplasma antigen

5. Urine Legionella antigen 


\section{Correct! \\ 3. Serum coccidiomycosis antibody}

The patient is being seen in Minnesota and thoracic infections differ between the Southwest and the Midwest. In the Southwest, especially Arizona and Southern California, coccidioidomycosis (Valley Fever) is very common but she has no history of travel to the Southwest. In contrast, histoplasmosis is very uncommon in the Southwest but more common in the Midwest. Similarly, Legionella is more common in the Midwest. The Legionella urinary antigen test is a relatively inexpensive, rapid and specific test for L. pneumophila serogroup 1 . However, it has a sensitivity of only about $70 \%$ and does not detect other serogroups. Influenza is common in both locales.

Her AFB smear, urine Legionella antigen, Blastomyces antibody, Cryptococcus antigen, and influenza PCR from nasopharynx swab were all negative. However, her urine histoplasma antigen was positive (> $39 \mathrm{mg} / \mathrm{mL})$.

Which of the following are appropriate to do next?

1. Bone marrow biopsy

2. Bronchoscopy with bronchoalveolar lavage

3. Examination of the peripheral blood smear for intracellular organisms

4. Histoplasma complement fixation and immunodiffusion

5. All of the above 


\section{Correct! \\ 5. All of the above.}

The urine and/or serum histoplasma antigen is an aid in the rapid diagnosis of disseminated or acute pulmonary histoplasmosis. Testing both urine and serum offers the highest sensitivity, as some patients may have a negative serum but a positive urine histoplasma antigen. False-positive and false-negative results do occur. Antigen results should be correlated with clinical and culture findings. Cross-reactions occur in blastomycosis and coccidioidomycosis.

The patient's Histoplasma complement fixation and immunodiffusion were negative. Bronchoscopy and bronchoalveolar lavage (BAL) had a negative fungal smear, but a blood smear showed intracellular fungal organisms. Surprisingly, her BAL fluid was positive for Pneumocystis jiroveci pneumonia (PCP) by PCR (strong positive per lab) although her PCP smear was negative.

Which of the following is appropriate to do next?

1. Begin antifungal therapy

2. Begin PCP therapy

3. HIV serology

4. 1 and 3

5. All of the above 


\section{Correct! \\ 5. All of the above}

Cultures grew Histoplasma capsulatum from the sputum, blood cultures, BAL and bone marrow biopsy. This patient has two infections (PCP and disseminated histoplasmosis) that are, with rare exceptions, only seen in immunocompromised patients. When such infections are seen, patients should be evaluated for immunocompromising conditions. Therefore, HIV serology and other tests of immunocompromise are indicated.

After discharge from the hospital, the patient was seen in the endocrinology clinic and ultimately diagnosed with Cushing's disease secondary to pituitary adenoma, which likely led to her immunosuppressed state and disseminated histoplasmosis and coinfection with PCP. The adenoma was surgically removed. She completed a course of trimethoprim/sulfamethoxazole and remains on itraconazole for the disseminated histoplasmosis and replacement doses of oral steroids.

Clinical Pearls (Useful information for care providers)

\section{Provider Pearls (Physicians/PAs/NPs)}

Histoplasmosis has been reported throughout the world, but Histoplasma capsulatum $(H$. capsulatum) is endemic to the Americas and some parts of Europe and Africa. Clinical cases are usually secondary to sporadic exposure, but outbreaks in areas of construction have been documented. $H$. capsulatum is a dimorphic fungus with a soilbased environmental reservoir and has been noted to thrive in areas with bird and bat excrement.

H. capsulatum causes clinical infection after inhalation. In the alveolar space, it is phagocytized by macrophages and via the macrophages is disseminated throughout the reticuloendothelial system. The immune response is dependent T-cell mediated response and multiple cytokines.

The majority of $H$. capsulatum infections are self-limiting with symptoms often confused with an acute viral syndrome or community-acquired pneumonia. Multiple clinical entities secondary to infection exist, although not all require antifungal treatment (although may require other supportive treatments). Traditionally, the entities requiring treatment include acute and pulmonary histoplasmosis, progressive disseminated histoplasmosis and mediastinal lymphadenitis.

Patients with reduced cellular immunity (e.g. AIDS, corticosteroids and extremes of age) are at risk for dissemination. Symptoms include, but are not limited to, fever, malaise, anorexia and weight loss. Physical exam may show signs of pancytopenias, cutaneous lesions or ulcerations, lymphadenopathy and hepatosplenomegaly. Laboratory findings may include pancytopenias, elevated alkaline phosphatase, LDH and inflammatory 
markers. The disease can involve any organ system, and therefore presentations can vary dramatically.

Diagnosis of $H$. capsulatum infection can be done via antigen detection, PCR assay, antibody tests, skin tests, biopsies and BAL. In acutely ill patients, biopsy and/or BAL (if appropriate) should be done as soon as possible.

Treatment for histoplasmosis varies based on type and severity of infection. Mild and moderate infections may be treated with oral itraconazole. For patients who are acutely ill Amphotericin B is the treatment of choice with transition to itraconazole once clinical improvement has occurred. Duration of treatment also varies, but is normally prolonged (months to years). Immunosuppressed patients with a history of disseminated histoplasmosis should receive secondary prophylaxis after completion of treatment until immune reconstitution.

Cushing's disease is responsible for about $70 \%$ of the cases of noniatrogenic Cushing's syndrome, but is responsible for less than $10 \%$ of related opportunistic infections.

Some of her presenting symptoms, such as the weakness and easy bruising may have also been related to the glucocorticoid excess. The immunosuppression secondary to glucocorticoid excess essentially affect all cell lines involved with immune and inflammatory responses, although depletion of CD4+ cells, cytokine dysregulation and suppression of neutrophil function are of significant concern when discussing the immune response to $H$. capsulatum.

\section{Nursing Pearls}

- Nursing would verify and initiate what kind of isolation is required for patient. Histoplasmosis and Pneumocystis jiroveci pneumonia (PCP) require standard precautions only (Standard Precautions provide the primary strategy for prevention and control of healthcare associated infections. They are to be routinely used in the care of all patients. Appropriate personal protective equipment must be used when exposure to blood and/or body fluids is anticipated.)

- Supportive care and symptom management as needed

- Psychosocial support for patient and family as needed

- Education on tests and procedures as well as patient disease/diagnosis as needed.

\section{Respiratory Therapy Pearls}

- Oxygen therapy as needed.

- The need for bronchial hygiene therapy should be assessed.

- Assessment should include:

o Ineffective cough, secretion retention, atelectasis

o Assist cough devices, vibratory PEP devices, or lung expansion therapy can be considered.

o If patient still has need for bronchial hygiene adjuncts upon dismissal, education on the devices needs to be done before dismissal. 


\section{Pharmacy Pearls}

- Amphotericin liposomal formulations are dosed at 3-5 mg/kg/day and generally the preferred product. If amphotericin deoxycholate is used, recommended dosing is $0.7-1.0 \mathrm{mg} / \mathrm{kg} / \mathrm{day}$.

- Oral Itraconazole:

o Administer a loading dose of $200 \mathrm{mg}$ three times daily for three days, then decrease to $200 \mathrm{mg}$ twice or once daily. No dose adjustment is needed for renal dysfunction.

o Itraconazole capsule absorption is acid dependent, give capsules with food and avoid acid suppressing medications. Itraconazole solution should be given on an empty stomach.

- Itraconazole has a long half life. Levels should be collected at seven days from initiation. Random levels are sufficient, goal serum level $>1 \mathrm{mcg} / \mathrm{mL}$ by HPLC method or $>3 \mathrm{mcg} / \mathrm{mL}$ by bioassay level. Combine itraconazole plus hydroxy-itraconazole metabolite for total level.

- Fluconazole is active against histoplasmosis but is not as effective and has resistant issues.

- Voriconazole has shown promise in treating histoplasmosis, but further clinical data is needed. The echinocandins do not have activity against histoplasmosis.

\section{References}

1. Stout JE, Yu VL. Legionellosis. N Engl J Med. 1997;337(10):682-7. [CrossRef] [PubMed]

2. Hauser PM, Bille J, Lass-Flörl C, Geltner C, Feldmesser M, Levi M, Patel H, Muggia V, Alexander B, Hughes M, Follett SA, Cui X, Leung F, Morgan G, Moody A, Perlin DS, Denning DW. Multicenter, prospective clinical evaluation of respiratory samples from subjects at risk for Pneumocystis jirovecii infection by use of a commercial realtime PCR assay. J Clin Microbiol. 2011;49(5):1872-8. [CrossRef] [PubMed]

3. Kauffman CA., Histoplasmosis: a clinical and laboratory update. Clin Microbiol Rev. 2007;20(1):115-32. [CrossRef] [PubMed]

4. Lionakis, M.S. and D.P. Kontoyiannis, Glucocorticoids and invasive fungal infections. Lancet. 2003;362(9398):1828-38. [CrossRef] [PubMed]

5. Kirk LF Jr, Hash RB, Katner HP, Jones T. Cushing's disease: clinical manifestations and diagnostic evaluation. Am Fam Physician. 2000:62(5):1119-27, 1133-4. [PubMed]

6. Knox, K.S. and C.A. Hage, Histoplasmosis. Proc Am Thorac Soc. 2010:7(3):169-72.

7. [CrossRef] [PubMed]

8. Kosseifi SG, Nassour DN, Shaikh MA, Sarubbi FA, Jordan RM, Peiris AN. Nodular pulmonary histoplasmosis in Cushing's disease: a case report and literature review. Tenn Med. 2007;100(12):44-6.[Pubmed]

9. Kadaria D, Muthiah MP, Sinclair SE. Unusual presentations of disseminated histoplasmosis. Tenn Med. 2012;105(3):39-40. [PubMed] 